\title{
Headache relevance in outpatient activity during Covid-19 pandemic
}

\author{
Francesca Gatti ${ }^{1}$ - Letizia Manneschi ${ }^{1}$
}

Published online: 27 August 2020

(C) Fondazione Società Italiana di Neurologia 2020

Nowadays headache disorders represent a great impact on the sanitary system, and in fact the WHO currently reports that the worldwide prevalence among adults of current headache disorder is estimated to be around 50\%; moreover, in Global Burden of Disease Study, headache disorders collectively were reported as the third highest cause of years lost due to disability. The most common headache disorders are migraine, tension-type headache (TTH) and trigeminal autonomic cephalalgias (TACs). Moreover, in many recent studies, headache is reported as one of the most frequent admitting symptoms to the emergency department, even if it has been well stated that the ED is a suboptimal place for headache treatment, suggesting that an urgent admission to a headache centre should be preferred even in the acute headache attacks [1].

At the beginning of the year 2020, with the spread of the Covid-19 pandemic, we observed major changes in the way we used to deliver primary care services: In fact, since March, many structures have been readdressed in SARS-COV-2 hospitals and all emergency department accesses were reserved for Covid-19 patients. Also, all non-urgent outpatient activities in Italy were suspended, meaning that headache centres could not deliver any service.

In this context, we would like to report our recent experience. Fidenza is a middle-sized town in northern Italy, in the third Italian region for total number of Covid-19 infection cases; the healthcare district of Fidenza gathers a total population of approximately 100,000 people and refers to the main hospital of Fidenza-Vaio. During the Covid-19 pandemic, this hospital was soon converted to a Covid-19only structure and the headache centre was closed, as well as all other neurologic outpatient activities. In this situation, a space dedicated only to urgent outpatient consults (up to 10 days) was created.

Francesca Gatti

francesca44gatti@gmail.com

1 Dipartimento Cure Primarie, Distretto di Fidenza, Ausl Parma, Via don E. Tincati, 5, 43036 Fidenza (PR), Italy
To assure maximum safety to all the patients and to prosecute the continuity of care, the use of telemedicine was soon implemented, including both phone calls and video calls, in order to provide assistance to the largest possible number of patients minimizing the hospital accesses.

The need for social distancing has been witnessed all over the world, and in fact some studies already report their successful experience in teleneurology, due to the urgent need to continue to deliver outpatient care, and focus on the fact that this use of instruments has the potential to fulfil the gap created by the pandemic crisis [2].

In our experience of neurological urgent outpatient activity during the Covid- 19 pandemic, in nearly 3 months, from 9 March to 20 May 2020, with a mean of 7.1 required consults per week in the whole district of Fidenza, the most frequent symptom reported was headache (38.8\%), followed by dizziness $(12.5 \%)$ and in the $5.6 \%$ of cases, these two symptoms were reported by the same patient.

Those patients were female in $64.5 \%$ of cases and male in $35.5 \%$, and the mean age was 47.2 , with a standard deviation of 18.8 .

Among our reported cases of headache and dizziness, $21.6 \%$ were first consults and $78.4 \%$ were follow-up or acute exacerbations.

Regarding the headache classification, we observed $42.3 \%$ migraines (of which $75 \%$ without aura and $25 \%$ with aura); tension-type headache was diagnosed in $7.1 \%$; regarding trigeminal autonomic cephalalgias, cluster headache represented $10.7 \%$ of cases and trigeminal neuralgia was observed in $7.1 \%$ of cases.

Moreover, the majority of consults was possible thanks to the use of telemedicine for a total of 39 consults $(54.2 \%)$.

Only 1 patient who referred headache resulted positive for SARS-COV-2, diagnosed with nasopharyngeal and oropharyngeal swab; all other patients were not tested, though we suspected a past infection in some cases.

We need to keep in mind that migraine and dizziness were also reported as Covid-19 symptoms in a $15 \%$ of patients in the main epidemiological meta-analysis. In particular, these 
studies reported neurological symptoms as frequent, though not highly specific; other related symptoms were impaired consciousness, anosmia, ageusia and meningeal signs [3].

The headache centre in Fidenza used to cover about 30 consults per month (considering 350 consults in the whole year 2019), both urgent and non-urgent; however, it is difficult to compare the previous activity with the neurological urgent outpatient activity that took place during the pandemic: The overall number of patients referring headache remained similar, but there has certainly been a great rise in urgent consults due to headache.

In conclusion, headache, associated with dizziness or not, was in our experience the most reported symptom requiring an urgent neurological consult in the first 2 months and a half of lockdown caused by Covid-19, thus representing a great increase in our context.

We could not acknowledge the exact number of SARSCOV-2 positive patients among our sample because of the limited indications to perform nasopharyngeal and oropharyngeal swab, in particular during the first months of the pandemic.

This elevated number of reported headaches could also be referred to the lifestyle changes required by the lockdown, leading to less social interaction, more sleep disorders and increase of stress. In particular, 3 patients in our sample reported specific alterations: One reported sleep deprivation due to frequent awakenings during the night, one other patient reported important mood deflection and the third one referred an increase in stress. However, we believe that those reported psychological impact could be just the tip of the iceberg considering that the recent social context completely altered the everyday routine of everyone.

Furthermore, from our experience, we believe that the use of instruments such as telemedicine will probably lead to a future change in our patients' treatment and follow-up, even outside the pandemic setting.

\section{Compliance with ethical standards}

Conflict of interest The authors declare that they have no conflict of interest.

Ethical approval All procedures performed in studies involving human participants were in accordance with the ethical standards of the institutional and/or national research committee and with the 1964 Helsinki declaration and its later amendments or comparable ethical standards. Informed consent was obtained from all individual participants included in the study.

\section{References}

1. Minen MT, Ortega E, Lipton RB, Cowan R (2018) American Headache Society survey about urgent and emergency management of headache patients. Headache. 58(9):1389-1396

2. McGinley MP, Ontaneda D, Wang Z, Weber M, Shook S, Stanton M, Bermel R (2020) Teleneurology as a solution for outpatient care during the COVID-19 pandemic. Telemed e-Health Online ahead of print: June 16, 2020

3. Carod-Artal FJ (2020) Neurological complications of coronavirus and COVID-19. Rev Neurol 70(9):311-322

Publisher's note Springer Nature remains neutral with regard to jurisdictional claims in published maps and institutional affiliations. 